\title{
Uterine Sarcoma - Difficult to Diagnose, Hard to Treat
}

\author{
Peter Mallmann \\ Klinik und Poliklinik für Frauenheilkunde und Geburtshilfe, Universitätsklinikum Köln, Cologne, Germany
}

Carcinosarcomas and other uterine sarcomas are rare neoplasms, with an annual world-wide incidence of between 0.5 and 3.3 cases per 100,000 women, accounting for fewer than 3-9\% of all cancers of the uterine corpus. Carcinosarcomas, the most common uterine sarcomas, are derived from the endometrium and demonstrate both epithelial and stromal differentiations. Nowadays, they are classified as endometrial carcinomas. $60-70 \%$ of all uterine sarcomas are leiomyosarcomas; undifferentiated uterine sarcomas, low-grade, and high-grade endometrial stromal sarcomas account for about $10 \%$, respectively. Each of these tumors has unique epidemiological as well as clinical and pathologic features.

Up to now, the major clinical challenge in the management of uterine sarcomas remains early diagnosis. As symptoms are unspecific, most cases are diagnosed as incidental findings after hysterectomy or morcellation of fibroids. The most frequent clinical symptoms are abnormal vaginal bleeding and an enlarging or fast growing uterus. A fast growing uterus in post-menopausal women and abnormal vaginal bleeding should prompt further evaluation. Unfortunately, the typical diagnostic procedure for diagnosing uterine sarcomas, endometrial sampling, has only low sensitivity. Symptomatic patients with uterine sarcomas receive a correct pre-operative diagnosis in less than $50 \%$ of all cases, which is probably related to the origin of these neoplasms in the myometrium. Furthermore, even if the correct diagnosis of a malignant disease is made, often only the epithelial component is evident from endometrial specimens and therefore very often the diagnosis of a poorly differentiated endometrial cancer is made, but not the correct diagnosis of uterine sarcoma. The typical diagnostic procedure in case of bleeding disorders, fractional dilatation and curettage, cannot exclude uterine sarcoma. In any case, rapidly enlarging leiomyoma, especially in post-menopausal women, necessitates further evaluation, usually by hysterectomy without morcellation. The most important diagnostic procedure is vaginal ultrasound and Doppler sonography. Unfortunately, neither ultrasound nor computer to- mography, magnetic resonance imaging, nor PET-CT are able to identify patients with uterine sarcomas. So the only way to exclude uterine sarcoma is the excision and pathologic examination.

The main problem in the management of the uterine sarcoma is the notable absence of data from randomized trials, both concerning the surgical and adjuvant treatment. In this issue of ONCOLOGY RESEARCH AND TREATMENT we will focus on 4 of the most common uterine sarcomas, uterine carcinosarcoma [1], uterine leiomyosarcoma [2], low-grade endometrial stromal sarcoma [3], and uterine adenosarcoma [4]. This mini-review series is based on the current S2k guideline of the working group of gynecologic oncology (Arbeitsgemeinschaft Gynäkologische Onkologie, AGO) and the German Cancer Society. The purpose of this review series is to present the current knowledge regarding specific problems in the diagnosis and therapy of uterine sarcomas. This series summarizes the clinical results of a structured consensus process being carried out by an interdisciplinary group of experts.

On behalf of the authors, I hope that this review series will help the reader in the daily practice in managing this disease.

\section{Disclosure Statement}

I declare that there is no conflict of interest.

\section{References}

1 Denschlag D, Ulrich UA: Urterine carcinosarcoma. Oncol Res Treat 2018;41 DOI $10.1159 / 000494335$

2 Juhasz-Böss I, Gabriel L, Bohle RM, Horn LC, Solomayer EF, Breitbach GP: Uterine leiomyosarcoma. Oncol Res Treat 2018;41 DOI: 10.1159/000494299.

3 Thiel FC, Halmen S: Low-grade endometrial stromal sarcoma - a review.Oncol Res Treat 2018;41 DOI: 10.1159/000494225.

4 Ulrich UA, Denschlag D: Uterine adenosarcoma. Oncol Res Treat 2018;41 DOI: $10.1159 / 000494067$.

\section{KARGER}

(c) 2018 S. Karger GmbH, Freiburg

Fax +497614520714 